PSICOLOGÍA

IBEROAMERICANA
Psicología Iberoamericana ISSN: 1405-0943

revista.psicologia@ibero.mx

Universidad Iberoamericana, Ciudad de México México

\title{
Adaptación y validación de la Escala del Modelo de Inversión en población mexicana
}

Escobar-Mota, Giovanna; Sánchez-Aragón, Rozzana; Lavalle, Daniel

Adaptación y validación de la Escala del Modelo de Inversión en población mexicana

Psicología Iberoamericana, vol. 27, núm. 1, 2019

Universidad Iberoamericana, Ciudad de México, México

Disponible en: http://www.redalyc.org/articulo.oa?id=133960951005 


\title{
Adaptación y validación de la Escala del Modelo de Inversión en población mexicana
}

\author{
Adaptation and validation of the Investment Model Scale in \\ the Mexican population \\ Giovanna Escobar-Mota gioesmota@gmail.com \\ Universidad Nacional Autónoma de México, México \\ DiD http://orcid.org/0000-0002-3665-137X \\ Rozzana Sánchez-Aragón \\ Universidad Nacional Autónoma de México, México \\ http://orcid.org/0000-0002-5952-8972 \\ Daniel Lavalle \\ Universidad Anáhuac México Norte, México \\ (D) http://orcid.org/0000-0002-6080-9210
}

Psicología Iberoamericana, vol. 27, núm. 1,2019

Universidad Iberoamericana, Ciudad de México, México

Recepción: 03 Mayo 2019 Aprobación: 24 Octubre 2019

Redalyc: http://www.redalyc.org/ articulo.oa?id=133960951005
Resumen: Existe una creciente preocupación por entender los factores asociados al mantenimiento y disolución de los vínculos afectivos, especialmente de las relaciones románticas. El modelo de Inversión propuesto inicialmente por Rusbult, Martz, y Agnew en 1998, ha demostrado ser de gran utilidad en la predicción de la estabilidad de diferentes tipos de relaciones. Así mismo, una cantidad creciente de estudios han utilizado la Escala del Modelo de Inversión en idioma inglés y no fue hasta 2012 que ésta se tradujo al idioma español en población chilena. Con base en la importancia de esta aproximación teórico-empírica y de la necesidad de contar con una versión de su medida aplicable al contexto mexicano, el propósito de esta investigación fue la validación en México de la Spanish Version of the Investment Model Scale de Vanderdrift (Vanderdrift, Agnew, \& Wilson, 2014) en adultos con pareja. La presente escala muestra la misma estructura de cuatro factores que la versión en inglés y la adaptación chilena que, en conjunto, explicaron 62.79\% de la varianza, y un Alpha de Chronbach de .73 , confirmando que la escala es confiable y válida y representa una herramienta útil en la medición de uno de los constructos de mayor relevancia en el entendimiento de las relaciones de pareja: el compromiso.

Palabras clave: compromiso, inversión, pareja, alternativas, satisfacción.

Abstract: There is a growing need to understand the factors associated with the maintenance and dissolution of emotional bonds, especially in romantic relationships. The Investment Model initially proposed by Rusbult et al. (1998) has proven to be useful in predicting the stability of different types of relationships. Likewise, an increasing number of studies have used the English Investment Model Scale and it was not until 2012 that it was translated into the Spanish language in the Chilean population. Based on the importance of this theoretical-empirical approach and the need to have a version of this instrument available to a Mexican audience, the purpose of this research was to validate the Spanish Version of the Investment Model Scale (Vanderdrift et al., 2014) in adults with a partner in the Mexican context. This scale shows the same four-factor structure as the English version and the Chilean adaptation. The scale showed $62.79 \%$ of the variance, and a Chronbach Alpha of .73, confirming that the scale is reliable and valid and represents a useful tool in the measuring the construct of commitment in understanding of intimate relationships.

Keywords: commitment, investment, couple, alternatives and satisfaction. 


\section{Introducción}

El estudio de los factores asociados al mantenimiento y duración de las relaciones de pareja ha sido objeto de estudio de diversas disciplinas incluidas la sociología, la filosofía y por ende la psicología desde distintas perspectivas y aplicaciones como lo son las áreas clínica y social (Alzugaray \& García, 2016).

Por muchos años, la duración del vínculo romántico o de pareja ha sido considerado uno de los principales indicadores de éxito en la cultura mexicana y de consecuencias negativas asociadas a su rompimiento (O’Connor, 1990; Ojeda \& Fagoaga, 2008; Sánchez Aragón \& Retana Franco, 2012). Por tal motivo, psicólogos sociales, estudiosos en el área de las relaciones de pareja, se han dado a la tarea de identificar las variables asociadas a mantener dicho vínculo, o bien, conocer el papel de ciertos aspectos individuales, sociales o contextuales en su disolución.

En la década de los noventas y a raíz de la creciente taza de divorcios en diferentes países del mundo -particularmente en América-, se observó un gran interés por publicar estudios respecto al entendimiento de la estabilidad y funcionalidad de la pareja y con ello la importancia de la noción de compromiso como factor de peso en ellos (Brehm, Miller, Perlman, \& Campbell, 2002). Otras variables como el tiempo en la relación (Ogolsky, 2009), satisfacción y personalidad (Spotts et al., 2005), fidelidad (Sirvent, 2011) y estrategias de mantenimiento (Nina-Estrella, 2011), comprobaron ser significativas para la estabilidad de la relación, contribuyendo a la permanencia dentro del vínculo, es decir, cada una de ellas, con sus propias características, aportan durante la interacción diádica un nivel mayor de explicación del fenómeno.

Por otro lado, este tipo de relaciones denominadas "de pareja", "románticas" o "íntimas", se caracterizan por periodos de estabilidad, pero también de inestabilidad propios de la vida en común, dando oportunidad a cada uno de sus miembros a evaluar tanto a su pareja como a la relación per se (Forgas \& Fitness, 2008). Es decir, cualquier relación que supera cierta temporalidad está sujeta a que sus miembros evalúen en términos de costos y beneficios, tanto a la pareja como a la relación en sí. Esta evaluación puede tener diversas consecuencias, entre ellas la reafirmación de que la decisión fue la correcta o la consciencia de alguna falta (Hoffman, Agnew, Lehmiller, \& Duncan, 2009).

A pesar de que existen muchos modelos teóricos e investigación empírica en procesos cognoscitivos y su influencia en la dinámica de las relaciones de pareja (Perlman \& Duck, 2006), existe un modelo teórico que permite la comprensión de los cambios en las relaciones a partir de aspectos centrales a su mantenimiento. Así, basado en nociones de la Teoría de la Interdependencia, Kelley y Thibaut (1978 en Forgas \& Fitness, 2008) y Rusbult et al. (1998) proponen un modelo para explicar los factores individuales y relacionales que subyacen al compromiso en una relación romántica. Esta autora propone que: 1) el nivel de satisfacción o los beneficios obtenidos en la relación; 2) la calidad de cada una de las alternativas, entendida como posibles beneficios que puede 
proporcionar una tercera persona y 3) el tamaño de la inversión hecha en la relación son importantes predictores de compromiso. Más tarde Goodfriend y Agnew (2008) reconceptualizan el factor de inversiones y lo extienden más allá de sus orígenes. Considerando que surge de la teoría de la Inversión y por lo tanto de una concepción económica, estos autores plantean que las inversiones van más allá de los recursos materiales; es decir, "las inversiones" incluyen cualquier pérdida en caso de que se termine la relación ya sea planes a futuro (reales o fantasiosos), hijos, pertenencias o incluso pensamientos idealizados tales como: "hasta que la muerte nos separe". La posibilidad latente de perder tales inversiones puede influir en la decisión de mantenerse dentro de la relación. Tomando en consideración estas aportaciones, se puede decir, entonces, que la inversión puede ser extrínseca o intrínseca, tangible o intangible; de tal forma que el modelo podría ser así: si la satisfacción obtenible por parte de una tercera persona es mayor que la obtenida en la relación principal, y las inversiones son bajas, el compromiso con la pareja actual se reducirá.

Siguiendo esta propuesta de que el compromiso es alimentado por tres factores independientes, Agnew, Arriaga y Wilson (2008) proponen el Modelo de Inversión del Compromiso en la Relación, mismo que proporciona una comprensión conceptual de los cambios que la gente hace de un tipo de relación a otra, y da cuenta tanto de la continuidad en las relaciones como de los cambios en las mismas.

Este modelo ha sido probado en numerosas investigaciones (Agnew, Arriaga, \& Wilson, 2008; Le, Dove, Agnew, Korn, \& Mutso, 2010; Warren, Harvey, \& Agnew, 2012) como, por ejemplo, un estudio en Madrid que examinó la capacidad del Modelo de Inversión para diferenciar entre individuos en relaciones estables a frente inestables, así como la capacidad de las variables del modelo (satisfacción matrimonial, inversión y relaciones alternativas) para predecir el compromiso y la estabilidad de las relaciones matrimoniales. Èste confirmó la relevancia de la satisfacción matrimonial para el compromiso con la relación, y de este último en la predicción de la estabilidad marital. Asimismo, la percepción de las relaciones alternativas como mejores que la actual, y no la disponibilidad a éstas alternativas, correlacionó negativa y significativamente con el compromiso (Íñigo, 2000).

Para probar la pertinencia del Modelo de Inversión del Compromiso en la Relación en distintos contextos culturales frente al que se acuñó o probó, se requiere de instrumentos de medición válidos y confiables para la población bajo estudio. Tal es el caso de la Escala del Modelo de Inversión, un instrumento diseñado para medir cuatro constructos: el compromiso, la satisfacción, la calidad de las alternativas y el tamaño de la inversión. Rusbult, Martz y Agnew (1998) un realizaron análisis de confiabilidad revelando buena consistencia interna entre los elementos diseñados para medir cada constructo. De esta manera, los análisis realizados revelaron la presencia de cuatro factores independientes. Así, la Escala del Modelo de Inversión demostró ser un predictor eficaz que mostró si el vínculo persistió o terminó. 
Posteriormente, (Vanderdrift et al., 2014) validaron la Escala del Modelo de Inversión en población hispana en Chile (Spanish Version of the Investment Model Scale), misma que ha generado información valiosa respecto a estudios que predicen la disolución-mantenimiento de los vínculos de pareja (Le et al., 2010), así como la persistencia asociada a comportamientos de sacrificio, interdependencia cognoscitiva y confianza (Wieselquist, Rusbult, Foster, \& Agnew, 1999).

Con base en la importancia de esta aproximación teórico-empírica y de la necesidad de contar con una versión de su medida aplicable a la población mexicana, el propósito de esta investigación encontrar, a través de su validación en adultos con pareja México, el modelo de inversión se manifiesta con la misma estructura factorial.

\section{Método}

Dado que los objetivos del presente estudio eran explorar cómo se manifiesta el modelo de inversión en población mexicana, se decidió realizar un análisis factorial exploratorio para tratar de encontrar o establecer, de manera exploratoria, una estructura interna, al generar nuevos factores a partir de un conjunto de variables, o reducir el número de éstas (Méndez Martínez, Alonso, \& Sepúlveda, 2012).

\section{Participantes}

Se trabajó con una muestra propositiva, no probabilística (Kerlinger, 1988), complementada por la técnica de muestreo llamada bola de nieve, que constó de 305 participantes (203 mujeres y 102 hombres), en un rango de edades entre los 18 a los 65 años $(M=26$ años, $\mathrm{DE}=9.8)$. Cabe señalar que el rango de edades fue definido a partir de la investigación original llevada a cabo por Rusbult et al. (1998) y el tamaño de la muestra se calculó a partir del número de reactivos; es decir, autores como Tabachnick y Fidell (2007) indican que por cada reactivo es necesario contar con cinco a diez participantes, por lo que la muestra en cuestión rebasa dicho lineamiento. Del total de la muestra, el 55\% de los participantes reportaron tener un grado escolar de licenciatura, seguido de preparatoria (17\%), y posgrado (11\%). El 68\% de los participantes eran solteros al momento del estudio; sin embargo, todos sostenían una relación de pareja al momento del estudio con un promedio de 5 años de duración en su relación. El 15\% se autodenominó con orientación no heterosexual, mientras que el $75 \%$ tenía una relación con orientación heterosexual, $75 \%$ de los participantes consideró su relación como exclusivamente monógama mientras que el $22 \%$ como no monógama.

\section{Instrumentos}

Escala del Modelo de Inversión, traducida y adaptada de la versión del idioma español validada en Chile por (Vanderdrift et al., 2014) que 
consta de 22 reactivos tipo Likert con 9 opciones de respuesta que indican grados de acuerdo con cada afirmación. Por medio de un análisis factorial exploratorio con rotación Promax que explicó el 62.79\% de varianza, se obtuvieron cuatro factores con valor propio mayor a uno: 1) Satisfacción: mide beneficios y satisfacción de necesidades afectivas $(\alpha=.87)$, consta de 5 reactivos como, por ejemplo, "Me siento satisfecho/ a con nuestra relación"; 2) Alternativas: mide el grado de deseabilidad percibida en la mejor opción disponible a la relación $(\alpha=.82)$, consta de 5 reactivos como: "Hay otras personas que me atraen mucho con las que puede que me involucre como pareja"; 3) Inversiones: mide la magnitud e importancia de los recursos asociados a la relación actual $(\alpha=.74)$, consta de 5 reactivos como, por ejemplo, "Muchos aspectos de mi vida han quedado ligados a mi pareja (actividades recreativas, etcétera.), y perdería todo eso si nos separamos"; y 4) Compromiso: mide la intención de persistir en la relación actual $(\alpha=.88)$, consta de 7 reactivos tales como "Deseo que nuestra relación dure para siempre". Dado que en la presente investigación se retomó la versión chilena, fue necesario el realizar algunas adaptaciones léxicas con el objetivo de emplear la variante del español mexicano como, por ejemplo, decía "He puesto mucho en nuestra relación, lo cual perdería si la relación se terminara” y se modificó: "He invertido mucho en mi relación con mi pareja, en comparación con otra gente que conozco". Cabe mencionar que la presente versión incluía únicamente cinco niveles de respuesta en la Escala Likert.

Además de la escala mencionada, a todos los participantes se les preguntaron datos sociodemográficos tales como edad, sexo, orientación sexual, tiempo en la relación y si consideraban su relación exclusivamente monógama o no monógama.

\section{Procedimiento}

Los participantes respondieron la escala de dos formas, en físico y en electrónico utilizando la plataforma Survey Monkey (www.surveymonkey.com). Esta decisión fue tomada con base en la idea de que se optimizaría de esta forma la aplicación en términos de tiempo, así como de confidencialidad principalmente para las personas en relaciones no monógamas, o bien, con una orientación homosexual. Todas las personas que aceptaron participar en la investigación respondieron de manera voluntaria, anónima y confidencial. Se les aclaró que los datos serían tratados de manera estadística y que, si durante la aplicación surgían dudas con respecto a cómo contestar algún instrumento, contarían con apoyo inmediatamente. El tiempo que les llevó responder fueron aproximadamente 15 minutos y la recolección de los datos duró aproximadamente dos meses. 
Con el fin de validar la Escala del Modelo de Inversión (Vanderdrift et al., 2014) se llevó a cabo el procedimiento propuesto por Reyes Lagunes \& García y Barragán (2008), basado(s) en los lineamientos de validación descritos por Nunnally y Bernstein (1994) y haciendo uso del Paquete Estadístico para Ciencias Sociales (Statistical Package for the Social Sciences, SPSS) versión 19 con el objetivo de obtener una escala de medición psicológica válida y confiable para la población mexicana. El procedimiento detallado consistió en las siguientes etapas: 1) análisis de frecuencias, reactivo por reactivo, para identificar errores en la base y estar seguros de que todas las opciones de respuesta hubieran sido utilizadas por los participantes; 2) prueba t de Student para muestras independientes, reactivo por reactivo, para estimar el poder discriminativo de éstos; 3 ) con los 21 reactivos, se corrió un análisis Alpha de Cronbach para evaluar la consistencia interna de la escala; 4) análisis de inter-correlaciones de los reactivos para determinar el tipo de rotación del Análisis Factorial; y 5) análisis descriptivos y Alpha de Cronbach para estimar la confiabilidad de cada factor.

Dicho procedimiento elimina la posibilidad de errores aleatorios, y considerando que nuestra pregunta de investigación es conocer si el modelo de inversión es aplicable a población mexicana por medio de crear una medida válida y confiable, es de fundamental importancia que el instrumento esté evaluando lo que dice medir, caso contrario se estaría operacionalizando incorrectamente el atributo, al no corresponder lo que realmente se observa, con lo que se cree.

\section{Resultados}

De los 22 reactivos, se encontró que 21 discriminaban y su consistencia interna total obtuvo un coeficiente de confiabilidad de $\alpha=.73$. Ningún reactivo cargó por arriba de .40 en diferentes factores como se puede apreciar en las cargas factoriales presentadas en la Tabla 1. Posteriormente se realizó un análisis factorial exploratorio de componentes principales con rotación ortogonal (tipo Varimax) dado que la ventaja principal de las rotaciones ortogonales es su simplicidad, ya que los pesos representan las correlaciones entre los factores y las variables (Méndez Martínez et al., 2012; Tabachnick \& Fidell, 2007). La intención fundamental al realizar una rotación es encontrar una estructura simple para averiguar si se conformaba de cuatro factores independientes como la versión chilena, aunado al hecho de que por estarse validando en otro contexto cultural se requiere examinar cuidadosamente el comportamiento de los reactivos. Así se obtuvo una estructura factorial compuesta por cuatro factores independientes que, en conjunto, explicaron $62.79 \%$ de la varianza $\left(\mathrm{KMO}=.90\right.$, prueba de esfericidad de Bartlett $\mathrm{X}^{2}=3300.958, \mathrm{gl}=210$, $\mathrm{p}=.000)($ ver Tabla 1$)$. 


\begin{tabular}{|c|c|c|c|c|}
\hline & & & & \\
\hline & \multicolumn{4}{|c|}{ Factor } \\
\hline & 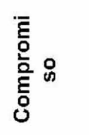 & 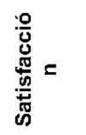 & 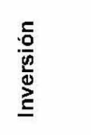 & 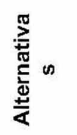 \\
\hline Reactivos & 6 & 5 & 5 & 5 \\
\hline Media & 3.76 & 3.70 & 2.97 & 2.77 \\
\hline Desviación Estándar & .91 & .79 & .78 & .83 \\
\hline Varianza factorial & .82 & .63 & .61 & .70 \\
\hline Alpha de Cronbach & .91 & .86 & .75 & .73 \\
\hline $\begin{array}{l}\text { 1. Estoy enfocado(a) en un futuro a largo plazo con mi relación (p. ej., me imagino } \\
\text { estando con mi pareja por varios años más). }\end{array}$ & .851 & & & \\
\hline 2. Deseo que nuestra relación dure para siempre. & .822 & & & \\
\hline 3. Deseo que nuestra relación dure mucho tiempo. & .772 & & & \\
\hline 4. Estoy comprometido(a) mantener la relación con mi pareja. & .723 & & & \\
\hline 5. Me siento fuertemente unido(a) mi pareja. & .717 & & & \\
\hline 6. Me siento muy apegado (a) nuestra relación. & .544 & & & \\
\hline 1. Me siento satisfecho (a) con nuestra relación. & & .766 & & \\
\hline 2. Nuestra relación me hace muy feliz. & & .759 & & \\
\hline 3. Mi relación es mucho mejor que las relaciones de otros. & & .752 & & \\
\hline 4. Mi relación es casi ideal. & & .701 & & \\
\hline $\begin{array}{l}\text { 5. Nuestra relación satisface bien mis necesidades de intimidad, compañerismo, } \\
\text { etc. }\end{array}$ & & .740 & & \\
\hline 1. He puesto mucho en nuestra relación, lo cual perdería si la relación se terminara. & & & .816 & \\
\hline $\begin{array}{l}\text { 2. Muchos aspectos de mi vida han quedado ligados a mi pareja (actividades } \\
\text { recreativas, etc.), y perdería todo eso si nos separáramos. }\end{array}$ & & & .757 & \\
\hline $\begin{array}{l}\text { 3. He invertido mucho en mi relación con mi pareja, en comparación con otra gente } \\
\text { que conozco. }\end{array}$ & & & .665 & \\
\hline $\begin{array}{l}\text { 4. Me siento muy involucrado/a en nuestra relación - como que le he dedicado } \\
\text { mucho. }\end{array}$ & & & .602 & \\
\hline $\begin{array}{l}\text { 5. Mis relaciones con amigos y familiares se complicarian si mi pareja y yo nos } \\
\text { separáramos (por ej., mi pareja es amigo/a de personas que me importan). }\end{array}$ & & & .559 & \\
\hline $\begin{array}{l}\text { 1. Si esta relación se acabara, yo estaría bien, pues encontraria fácilmente otra } \\
\text { pareja atractiva. }\end{array}$ & & & & .816 \\
\hline $\begin{array}{l}\text { 2. Tengo excelentes alternativas en lugar de esta relación (otra pareja, salir con mis } \\
\text { amigos/as, salir con ex parejas etc.). }\end{array}$ & & & & .788 \\
\hline $\begin{array}{l}\text { 3. Mis necesidades de intimidad, compañerismo, etc., podrian ser fácilmente } \\
\text { satisfechas en una relación alternativa con otra persona. }\end{array}$ & & & & .654 \\
\hline 4. Si nuestra relación terminara en un futuro cercano, no me afectaría mucho. & & & & .531 \\
\hline 5. Es probable que tenga una pareja diferente dentro del próximo año. & & & & .509 \\
\hline
\end{tabular}

\section{Discusión}

El propósito de esta investigación fue la validación en México de la Spanish Version of the Investment Model Scale de Vanderdrift et al. (2012) en adultos con pareja para lo cual se llevó a cabo el procedimiento dictado por Reyes Lagunes y García y Barragán (2008) que se indica para lograr medidas válidas y confiables con sensibilidad cultural. 
Lo hallazgos muestran que la versión obtenida en este estudio es una escala válida y confiable aplicable a muestras de personas mexicanas, adultas con pareja, ya que presenta características psicométricas robustas; es decir, se logró identificar una estructura de cuatro factores claros e independientes cuyos coeficientes de confiabilidad Alpha de Cronbach fueron apropiados (ver Tabla 2). Así los factores se definen de la siguiente manera:

El primer factor se denominó Compromiso y se refiere al deseo de mantener la relación de pareja por largo tiempo, así como enfocarse en dicha meta pues ello contribuye al sentimiento de unión y apego con el otro. Este factor se apoya en la literatura que indica que el compromiso es la decisión de formar una relación (Márquez Domínguez, 2010), la convicción de la pareja es la persona con la que se quiere vivir, pues es una etapa en la que se toman las cosas con más formalidad, guiando a futuro, el deseo de formar una familia (González \& García, 2009). Asimismo, el compromiso es considerado la parte fundamental de la existencia humana, depende de la decisión voluntaria y del peso que la sociedad le confiere a la relación de pareja (Rodríguez Salazar, 2001).

El segundo factor se llamó Satisfacción y se refiere a la medida en que la relación de pareja complace las necesidades de intimidad, compañerismo y hace feliz a la persona. La relevancia de este factor descansa en que precisamente la satisfacción, definida como la evaluación subjetiva que se tiene de la pareja y de la propia relación en términos de diversos aspectos, resulta ser el principal indicador de felicidad, éxito y calidad de dicho vínculo (Armenta-Hurtarte, Sánchez-Aragón, \& Díaz-Loving, 2012).

Por su parte el tercer factor titulado Inversión, se refiere a aquellos aspectos que han sido contribuciones personales a la relación como amigos, deudas, tiempo y bienes materiales que se verían amenazados si el vínculo terminara. $\mathrm{Al}$ respecto, se debe decir que es natural que en un vínculo romántico se vayan depositando "valores" no solo económicos, sino psicológicos o sociales que van fortaleciendo la relación ya que contribuyen a la construcción de ésta, del valor que se le confiere y del dolor que implicaría un rompimiento y con ello la factible pérdida de la inversión realizada (Hinde, 2007). Asimismo, autores como Lund (1985) señalan que las inversiones involucran acciones sobre las cuales las personas tienen control, y que el efecto del involucramiento conductual es crear un cambio cognoscitivo para que las expectativas acerca del futuro de la relación sean reforzadas.

Finalmente, el cuarto factor nombrado Alternativas, se refiere a la percepción que tiene la persona sobre las opciones posibles de personas que pudieran satisfacer las necesidades de intimidad y compañerismo de no contar con su pareja. Aunque en este caso la operacionalización incluye únicamente como alternativas a otras personas, la definición teórica del modelo contempla como alternativas al grado en el cual el individuo ve que sus necesidades importantes podrían ser satisfechas fuera de la relación, y no necesariamente ello implica a otra relación de pareja (Rusbult et al., 1998) como podría ser una actividad, trabajo, amigos, familia, etcétera. Hay que señalar que la percepción de alternativas 
está también influenciada por características personales, es decir, se ha encontrado que las personas con alta auto-estima y aquellas con una fuerte necesidad de autonomía, puede estar más preparadas a dejar una relación y encontrar otras opciones para sentirse satisfechos en su vida (De la Villa, García, Cuetos, \& Sirvent, 2017).

Ya contando con la versión válida y confiable de la Escala del Modelo de Inversión validada en la presente investigación, es posible el comparar los hallazgos de esta investigación con las contribuciones realizadas por (Rusbult et al., 1998) en población estadounidense y la realizada en población chilena (Vanderdrift et al., 2014). Primero que nada, se observó que las tres versiones resultaron muy parecidas ya que mostraron la obtención de los mismos factores: compromiso, satisfacción, alternativas e inversión. No obstante, estas similitudes, hay algunos detalles que variaron (ver Tabla 2).

Tabla 2

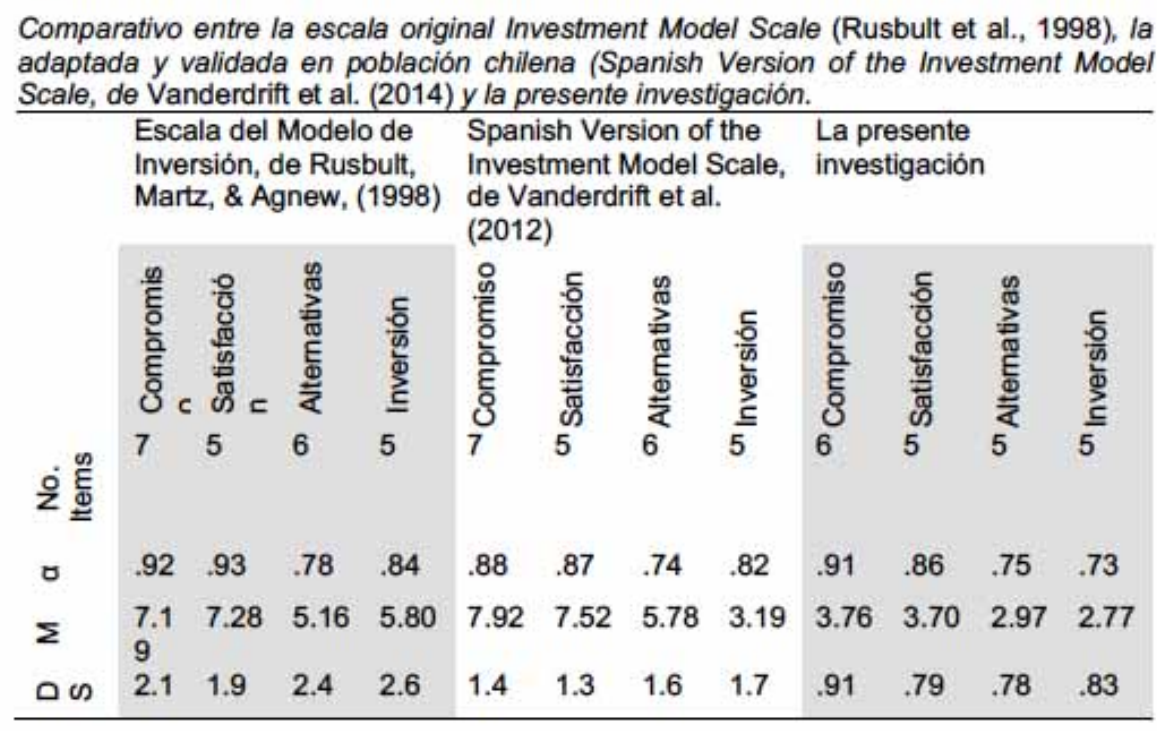

Si bien las tres escalas cumplen con los criterios de confiabilidad presentando Alphas de Chronbach superiores al criterio de .70 (Tabachnick \& Fidell, 2007), también muestran un patrón similar en el factor de Alternativas, ya que los tres casos muestran alphas bajas; esto puede deberse a que quienes se encuentran dentro de una relación, no tienen presente terminarla (Nina-Estrella, 2011).

Por otro lado, las personas están arriba de la media, lo cual indica que los ítems son entendibles y que las personas lograron fácilmente interpretarlo. Así mismo, las medias difieren debido a que la escala de la presente investigación utilizó cinco niveles de respuesta y no nueve como en la original y en la versión traducida para población chilena, sin embargo, también muestra un patrón coincidente en que tanto Compromiso como Satisfacción es más alto que Alternativas e Inversión, para las tres escalas. Esto puede deberse a que las personas tienen mayor claridad en lo que esperan en una relación que en lo que está en juego o podría sustituirla (Vangelisti, Reis, \& Fitzpatrick, 2004). Finalmente, la 
diferencia en las desviaciones estándar muestra una menor dispersión de las respuestas respecto a las medias en la escala de la presente investigación, sugiriendo una posible cohesión social en el entendimiento de lo que implica un compromiso con la pareja (Díaz Loving, 1999).

Por otro lado, las versiones anteriores de la escala fueron aplicadas en muestras conformadas por estudiantes de dos culturas distintas a la mexicana: la estadounidense y la chilena. La muestra empleada en este estudio no se limita a estudiantes sino que incluye adultos con un amplio rango de edad, distintas orientaciones sexuales y con relaciones de pareja de diversa índole y duración. Si bien en la Ciudad de México el grado promedio de escolaridad de la población de 15 años y más es de 11.1, lo que equivale a segundo año de educación media superior, es la entidad que tiene el promedio de escolaridad más alto del país, con 10.5 años, superando en casi dos grados al promedio nacional (INEGI, 2015). La investigación psicosocial de las relaciones íntimas debe apuntar a muestras más diversas y así contrarrestar el fenómeno de la normatividad en su estudio, arrojando hallazgos sesgados por ésta.

Así, el compromiso es el constructo psicológico que predomina en el comportamiento de la vida cotidiana de la pareja, así como la experiencia psicológica que experimenta en torno a "lo que implica" (Adams \& Jones, 1999; Duncombe, Harrison, Allan, \& Marsden, 2004; Impett, Beals, \& Peplau, 2001; Rhoades, Stanley, \& Markman, 2010). Por tal razón, el compromiso como constructo psicológico muestra ser un excelente indicador del nivel de ajuste de una relación a sí misma, así como un importante predictor de la permanencia en la relación (Holman, 2002; Le et al., 2010). Dado lo anterior, la validación de la presente escala representa una herramienta metodológica respaldada por una teoría sólida, como es la de la interdependencia, y por una vasta cantidad de estudios empíricos sujetos a replica en nuestra población.

En conclusión y con base en los factores identificados en esta investigación, los mexicanos identifican claramente la diferencia entre la satisfacción que brinda una relación de pareja, las inversiones a la misma, las alternativas a ésta y así se manifiesta el compromiso a mantenerse dentro de ella. El procedimiento estadístico empleado confirma que la Escala del Modelo de Inversión es confiable y válida y representa una herramienta útil en la medición de uno de los constructos de mayor relevancia en el entendimiento de las relaciones de pareja: el compromiso.

\section{Limitaciones}

Deben señalarse algunas de las limitaciones que se han detectado en el presente estudio como contar con una muestra muy específica y excesivamente homogénea en cuanto a características como nivel de escolaridad, diversas orientaciones sexuales y acuerdos de exclusividad en la pareja, como lo son las parejas no-monógamas. Ello significa que los datos solo pueden generalizarse a población de características similares. En futuras investigaciones sería conveniente incluir en la muestra otros perfiles sociodemográficos. 


\section{Conclusiones}

La presente escala será útil para futuros estudios sobre compromiso y mantenimiento de la relación de pareja e incluso para encontrar relaciones con otras variables de peso. Contar con escalas de medición que estén respaldadas por modelos teóricos estables y que a su vez se encuentren validadas en población mexicana permite, entre otras ventajas, el estudio de las diferencias culturales que pueden afectar a los procesos en las relaciones de pareja (Vanderdrift et al., 2014). Se espera que la existencia de un instrumento de medición válido y confiable que respalde el modelo de inversión promueva aún más la investigación sobre el compromiso y la interdependencia con la satisfacción, las inversiones y las alternativas, en las relaciones de pareja.

\section{Referencias}

Adams, J. M., \& Jones, W. H. (1999). Handbook of interpersonal commitment and relationship stability. Nueva York, NY, US: Kluwver Academic.

Agnew, C. R., Arriaga, X. B., \& Wilson, J. E. (2008). Committed to what? Using the bases of relational commitment model to understand continuity and change in social relationships. En J. Forgas \& J. Fitness (Eds.), Social relationships: Cognitive, affective, and motivational processes (pp. 147161). Nueva York, NY, US: Taylor \& Francis Group.

Alzugaray, C., \& García, F. (2016). Relaciones de pareja y bienestar psicológico. En M. A. Bilbao, D. Páez \& J. C. Oyanedel (Eds.), La felicidad de los chilenos: Estudios sobre bienestar (pp. 237-251). Santiago, Chile: USACH RiL editores.

Armenta-Hurtarte, C., Sánchez-Aragón, R., \& Díaz-Loving, R. (2012). ¿De qué manera el contexto afecta la satisfacción con la pareja? Suma Psicologica, 19(2), 51-62.

Brehm, S., Miller, R., Perlman, D., \& Campbell, S. (2002). Intimate relationships. Social influence. Nueva York, NY, US: McGraw-Hill.

De la Villa, M., García, A., Cuetos, G., \& Sirvent, C. (2017). Violencia en el noviazgo, dependencia emocional y autoestima en adolescentes y jóvenes españoles. Revista Iberoamericana de Psicología y Salud, 8(2), 96-107. htt ps://doi.org/10.23923/j.rips.2017.08.009

Díaz Loving, R. (Ed.). (1999). Antologíapsicosocial de lapareja. CDMX, México: Porrúa.

Duncombe, J., Harrison, K., Allan, G., \& Marsden, D. (Eds.). (2014). The state of affairs: Explorations in infidelity and commitment. Nueva York, NY, US: Psychology Press.

Forgas, J., \& Fitness, J. (2008). Social relationships: Cognitive, affective, and motivational processes. Nueva York, NY, US: Psychology Press.

González, T. T., \& García, A. O. (2009). El compromiso y la estabilidad en la pareja: Definición y dimensiones dentro de la población mexicana. Psicología Iberoamericana, 17(1), 38-47.

Goodfriend, W., \& Agnew, C. R. (2008). Sunken costs and desired plans: Examining different types of investments in close relationships. 
Personality and Social Psychology Bulletin, 34(12), 1639-1652. https://do i.org/10.1177/0146167208323743

Hinde, R. (2007). Bending the rules. The flexibility of absolutes in modern life. Nueva York, NY, US: Oxford University Press.

Hoffman, A. M., Agnew, C. R., Lehmiller, J. J., \& Duncan, N. T. (2009). Satisfaction, alternatives, investments, and the microfoundations of audience cost models. International Interactions, 35(4), 365-389. https:/ /doi.org/10.1080/03050620903328274

Holman, T. B. (2002). Premarital prediction of marital quality or breakup. Research theory, and practice. Nueva York, NY, US: Kluwer Academic Publishers.

Impett, E. A., Beals, K. P., \& Peplau, L. A. (2001). Testing the investment model of relationship commitment and stability in a longitudinal study of married couples. Current Psychology, 20(4), 312-326. https://doi.org/10. 1007/s12144-001-1014-3

Instituto Nacional de Estadístaca y Geografía (INEGI). (2015). Encuesta Intercensal 2015. Recuperado de https://www.inegi.org.mx/contenidos/ programas/intercensal/2015/doc/eic_2015_presentacion.pdf

Íñigo, D. M. (2000). Contrastación del modelo de inversión de Rusbult en una muestra de casados y divorciados. Psicothema, 12(1), 65-69.

Kerlinger, F. N. (1988). Investigación del comportamiento [Foundations of behavioral research] ( $2^{\mathrm{a}}$ ed.). Ciudad de México, Mexico: McGraw-Hill.

Le, B., Dove, N. L., Agnew, C. R., Korn, M. S., \& Mutso, A. A. (2010). Predicting nonmarital romantic relationship dissolution: A meta-analytic synthesis. Personal Relationships, 17(3), 377-390. https://doi.org/10.1111/j.14756811.2010.01285.x

Lund, M. (1985). The development of investment and commitment scales for predicting continuity of personal relationships. Journal of Social and Personal Relationships, 2(1), 3-23. https://doi.org/10.1177/026540758 5021001

Márquez Domínguez, J. F. (2010). Apego, auto-esquema y cultura, como determinantes del compromiso y la satisfacción dentro de las relaciones de pareja (Tesis doctoral). Universidad Nacional Autónoma de México, CDMX, México.

Méndez Martínez, C., Alonso, M., \& Sepúlveda, R. (2012). Introducción al análisis factorial exploratorio. Revista Colombiana de Psiquiatría, 41(1), 197-207.

Nina-Estrella, R. (2011). ¿Qué nos mantiene juntos? Explorando el compromiso y las estrategias de mantenimiento en la relación marital. Revista Intercontinental de Psicología y Educación, 13(2), 2197-2220.

Nunnally, J. C., \& Bernstein, I. H. (1994). Psychometric theory ( $3^{\mathrm{a}}$ ed.). Ann Arbour, MI, US: McGraw-Hill.

O'Connor, N. (1990). Déjalos ir con amor: La aceptación del duelo. CDMX, México: Trillas.

Ogolsky, B. G. (2009). Deconstructing the association between relationship maintenance and commitment: Testing two competing models. Personal Relationships, 16(1), 99-115. https://doi.org/10.1111/j.1475-6811.200 9.01212.x 
Ojeda, N., \& Fagoaga, G. (2008). Divorcio y separación conyugal en México en los albores del siglo. Revista Mexicana de Sociología, 70(1), 111-145.

Perlman, D., \& Duck, S. (1985). The seven seas of the study of personal relationships: From "the thousand islands" to interconnected waterways. En A. L. Vangelisti \& D. Perlman (Eds.), The Cambridge handbook of personal relationships (pp. 11-34). Cambridge, UK: Cambridge University Press. http://dx.doi.org/10.1017/CBO9780511606632.003

Reyes Lagunes, I., y García y Barragán, L. F. (2008). Procedimiento de validación psicométrica culturalmente relevante. La Psicología Social En México, XII, 625-630.

Rhoades, G. K., Stanley, S. M., \& Markman, H. J. (2010). Should I stay or should I go? Predicting dating relationship stability from four aspects of commitment. Journal of Family Psychology, 24(5), 543-550. https://doi. org/10.1037/a0021008

Rodríguez Salazar, T. (2001). Las razones del matrimonio, representaciones, relatos de vida y sociedad. Guadalajara, México: Universidad de Guadalajara.

Rusbult, C. E., Martz, O. J. M., \& Agnew, C. R. (1998). The Investment Model Scale\#: Measuring commitment level, satisfaction level, quality of alternatives, and investment size. Personal Relationships, 5(4), 357-391. h ttps://doi.org/10.1111/j.1475-6811.1998.tb00177.x

Sánchez Aragón, R., \& Retana Franco, B. E. (2012). Evaluación tridimensional del duelo amoroso en México. Revista Iberoamericana de Diagnóstico y Evaluación-e Avaliação Psicológica, 2(36), 49-69.

Sirvent, C. (2011). Fidelidad y compromiso en la relación de pareja (El trinomio fidelidad, compromiso y monogamia). Norte de Salud Mental, IX(40), 5771.

Spotts, E. L., Lichtenstein, P., Pedersen, N., Neiderhiser, J. M., Hansson, K., Cederblad, M., \& Reiss, D. (2005). Personality and marital satisfaction: A behavioural genetic analysis. European Journal of Personality, 19(3), 205227. https://doi.org/10.1002/per.545

Tabachnick, B. G., \& Fidell, L. S. (2007). Using multivariate statistics ( $5^{\mathrm{a}}$ ed.). Boston, MA, US: Pearson. https://doi.org/10.1037/022267

Vanderdrift, L. E., Agnew, C. R., \& Wilson, J. E. (2014). Spanish version of the Investment Model Scale. Personal Relationships, 21(1), 110-124. https:/ /doi.org/10.1111/pere.12016

Vangelisti, A., Reis, H., \& Fitzpatrick, M. A. (Eds.). (2004). Stability and change In relationships. Cambridge, UK: Cambridge University Press.

Warren, J. T., Harvey, S. M., \& Agnew, C. R. (2012). One love: Explicit monogamy agreements among heterosexual young adult couples at increased risk of sexually transmitted infections. Journal of Sex Research, 49(2-3), 282-289. https://doi.org/10.1080/00224499.2010.541952

Wieselquist, J., Rusbult, C. E., Foster, C. A., \& Agnew, C. R. (1999). Commitment, pro-relationship behavior, and trust in close relationships. Journal of Personality and Social Psychology, 77(5), 942-966. http://dx.d oi.org/10.1037/0022-3514.77.5.942 


\section{Notas}

[1] Edificio C, Primer piso, Cubículo 98, Facultad de Psicología, Universidad Nacional Autónoma de México; Avenida Universidad 3004, Col. Ciudad Universitaria, C.P. 04510, Delegación Coyoacán, Ciudad de México, México. Correo electrónico: gioesmota@gmail.com 